\title{
Estudo do desempenho elétrico e da composição química de baterias de Ni-HM original e falsificada
}

\section{Electrical performance and chemical composition studies on original and falsified Ni-MH batteries}

\author{
Alexandre Urbano ${ }^{1}$; Jair Scarminio ${ }^{1}$; Carlos Roberto Appoloni ${ }^{1}$; \\ Ricardo Floriano ${ }^{2}$; Fábio Luiz Melquíades ${ }^{3}$; Adenilson Oliveira dos Santos ${ }^{4}$; \\ Paulo Rogério Catarini ${ }^{5}$; Fábio Lopes ${ }^{5}$; Rafael Bonacin ${ }^{6}$
}

\section{Resumo}

Neste trabalho mostramos que a falsificação de produtos tecnológicos atinge também as baterias recarregáveis de níquel hidreto metálico (Ni-HM). Investigamos o desempenho elétrico e a composição química dos eletrodos de baterias de Ni-HM de formato AAA, originais e falsificadas, comercializadas em Londrina, Norte do Paraná. O desempenho elétrico das baterias foi quantificado medindo-se sua capacidade de carga, sob taxa de $0,2 \mathrm{C}$, e a sua potência elétrica média, nas taxas de 0,2 e 0,8 C. Para as análises da composição química as baterias foram desmontadas em vácuo, e seus eletrodos analisados por Fluorescência de Raios-X por Dispersão em Energia (EDXRF) e Difração de Raios-X (XRD). Observou-se que a capacidade de carga da bateria original foi de $920 \mathrm{mAh}$ contra $155 \mathrm{mAh}$ da falsificada, e as potências médias foram respectivamente $210 \mathrm{~mW}$ e $41 \mathrm{~mW}$. A composição química dos catodos das baterias, original e falsificadas, é de hidróxido de níquel $\left(\mathrm{Ni}(\mathrm{OH})_{2}\right)$. Os anodos, no entanto, são diferentes. Na original encontrou-se o composto $\mathrm{LaNi}_{5}$ e na falsificada o hidróxido de cádmio $(\mathrm{Cd}$ $\left.(\mathrm{OH})_{2}\right)$. As baterias originais, portanto, exibem 6 vezes mais capacidade de carga, 5 vezes mais potência a $0,2 \mathrm{C}$ e 6 vezes a $0,8 \mathrm{C}$, e ainda são menos agressivas ao meio ambiente por não conterem cádmio.

Palavras-chave: Baterias falsificadas. Níquel hidreto metálico. Cádmio.

\begin{abstract}
We show in this paper that falsifications on technological products have hit even rechargeable nickel metal hydride batteries (Ni-MH). The electrical performance and the electrode chemical composition were investigated for authentic and falsified AAA Ni-MH batteries, purchased in the Londrina market, Paraná State. Battery charge capacities were measured at 0,2 C discharge rate and average electrical power was measured at 0.2 and $0.8 \mathrm{C}$ discharge rate. To perform chemical composition analysis, the batteries were vacuum dismantled and their electrodes were characterized by Energy Dispersive X-Ray Fluorescence (EDXRF) and X-Ray Diffraction (XRD) techniques. It was observed that the charge capacities for the authentic and falsified batteries were 920 and $154 \mathrm{mAh}$, respectively. The average electrical powers were $210 \mathrm{~mW}$ for authentic and $41 \mathrm{~mW}$ for falsified batteries. The cathode chemical

1 Docentes do Departamento de Física da Universidade de Londrina (UEL).E-mail: aurbano@uel.br; scarmini@uel.br; appoloni@

2 Pós Graduando em Física do Departamento de Física da Universidade Estadual de Londrina (UEL). E-mail: ricardo_fisica@,

3 Docente do Departamento de Física da Universidade Estadual do Centro-Oeste (UNICENTRO). E-mail: fmelquiades@unicentro.br

4 Docente da Universidade Federal do Maranhão (UFMA). E-mail: adenilson1@gmail.com

5 Físicos MSc. do Departamento de Física da Universidade Estadual de Londrina (UEL). E-mail: prcsilva@uel.br; bonn@uel.br

${ }^{6}$ Graduando em Física do Departamento de Física da Universidade Estadual de Londrina (UEL). E-mail: rbonacindeoliveira@
\end{abstract} uel.br hotmail.com gmail.com 
composition was nickel hydroxide, $\left(\mathrm{Ni}(\mathrm{OH})_{2}\right)$, for both kinds of batteries. However, the anodes of these batteries were not composed by the same materials. The alloy $\mathrm{LaNi}_{5}$ was identified as the electroactive compound in the anode of the authentic battery, while cadmium hydroxide compound, $\left(\mathrm{Cd}(\mathrm{OH})_{2}\right)$, was identified in the falsified battery anode. The authentic battery therefore presented six times more charge capacity, five times more power at $0.2 \mathrm{C}$ discharge rate and 6 times at $0.8 \mathrm{C}$ than the falsified battery, and are yet less dangerous to environment due cadmium absence.

Key-words: Falsified batteries. Nickel metal hydride. Cadmium.

\section{Introdução}

As baterias de níquel hidreto metálico (NiHM) dominam uma fatia considerável do mercado de baterias recarregáveis por equiparem diversos aparelhos eletrônicos portáteis como tocadores de música (MP3, MP4), máquinas fotográficas, filmadoras, ferramentas portáteis, controles remotos, brinquedos, rádios, entre outros. Parte deste mercado é composto por baterias falsificadas, que segundo a Associação Brasileira da Indústria Elétrica e Eletrônica (COSTA, 2009), chega a atingir $30 \%$ do total das baterias comercializadas, estimulado principalmente pelos baixos preços das baterias falsificadas (AZAR; HOLMBERG; LINDGREN, 1996;).

A dificuldade na diferenciação entre baterias originais e falsificadas não é exclusividade apenas dos consumidores, mas também dos comerciantes. Para algumas marcas o processo de selagem do invólucro da bateria pode ser uma sensível característica. Nas baterias originais mais modernas o invólucro metálico é selado com solda TIG (Tugsten Inert Gas), enquanto que nas baterias falsificadas exibem o antigo selamento por estrangulamento (“clamping”) (BUCHMANN, 2001). Outras características, que podem ser observadas visualmente, são o acabamento das embalagens e dos rótulos e as suas tonalidades de cores. Dados obtidos em sítios da internet (mas sem comprovação científica) apontam que as baterias falsificadas de Ni-HM contêm cádmio. Se comprovado, este fato pode ser um grande problema do ponto de vista ambiental, visto que as baterias de Ni-HM podem legalmente ser descartadas no lixo doméstico, segundo a Resolução 257 de 1999 do Conselho Nacional do Meio Ambiente (1999) (AZAR; HOLMBERG; LINDGREN, 1996; RYDH; BOSVARD, 2003).

As baterias de Ni-HM emergiram justamente da necessidade de se substituir o cádmio, presente nas baterias de níquel-cádmio (Ni-Cd) (FENGA; GENGA; NORTHWOODB, 2001; MORIOKAA; NARUKAWAB; ITOUB, 2001; YUICHI; SHIGEO; KOICHI, 2001; OHMS et al., 2002). As baterias de Ni-HM são compostas basicamente por um catodo de hidróxido de níquel $\left(\mathrm{Ni}(\mathrm{OH})_{2}\right.$ ou $\mathrm{H}_{2} \mathrm{NiO}_{2}$ ), um separador de celulose, que é embebido pelo eletrólito de hidróxido de potássio dissolvido em água, e um anodo de hidreto metálico HM, onde $\mathrm{M}=\mathrm{LaNi}_{5}$ (SAKAI; UEHARA; ISHIKAWA, 1999; YAN; CUI, 1999; GENG et al., 2001). Historicamente, o anodo de hidreto metálico foi o composto que permitiu a substituição do cádmio nas baterias de $\mathrm{Ni}-\mathrm{Cd}$, devido às suas propriedades de intercalação do íon hidrogênio $\left(\mathrm{H}^{+}\right)$, que é o íon ativo nas baterias alcalinas de intercalação iônica (HUGGINS et al., 1994; SHUKLAA; VENUGOPALANB; HARIPRAKASHA, 2001). As reações parciais que descrevem os processos de carga e descarga dos eletrodos das baterias de NiHM são descritas abaixo.

$$
\begin{array}{lll}
\mathrm{H}_{2} \mathrm{NiO}_{2} \underset{\text { descarga }}{\stackrel{\text { carga }}{\rightleftarrows}} \mathrm{HNiO}_{2}+\mathrm{H}^{+}+\mathrm{e}^{-} & E^{0}=0,52 \mathrm{~V} \\
\mathrm{LaNi}_{5}+6 \mathrm{H}^{+}+6 \mathrm{e}^{-} \underset{\text { descarga }}{\rightleftarrows} & \mathrm{H}_{6} \mathrm{LaNi}_{5} & E^{0}=-0,83 \mathrm{~V}
\end{array}
$$

A motivação deste trabalho surgiu ao se confrontar as características impressas nos rótulos de baterias de Ni-HM originais e falsificadas, encontradas no comércio da cidade de Londrina - PR. Observouse, imediatamente, uma inconsistência grosseira nos 
valores apresentados para a capacidade de carga. Para o formato AAA (diâmetro $=10 \mathrm{~mm}$, altura $=$ 44,5 $\mathrm{mm}$ ) o valor informado para a capacidade de carga da bateria original era de $900 \mathrm{mAh}$, e para a falsificada o valor era de $3000 \mathrm{mAh}$. A tensão fornecida no rótulo era de $1,2 \mathrm{~V}$ para ambas. O preço das baterias variava de $\mathrm{R} \$ 15,00$ para a original e $\mathrm{R} \$ 5,00$ para a falsificada. Na análise de custo $v s$. benefício por capacidade de carga, levando em conta apenas as informações das embalagens, ficava evidente uma suposta superioridade da bateria falsificada. O valor em reais, de cada unidade de carga (Ah), era aproximadamente igual a 1,6 R $\$ / A h$, para a falsificada, contra $16 \mathrm{R} \$ / \mathrm{Ah}$, para a original.

O objetivo deste trabalho é a determinação do custo vs. benefício por capacidade de carga real das baterias. Para isso o desempenho elétrico das baterias de Ni-HM, originais e falsificadas, e as composições elementares dos seus eletrodos, foram caracterizados com o objetivo de se certificar suas reais condições frente àquelas informadas nas respectivas embalagens.

\section{Experimental}

Baterias recarregáveis de Ni-HM do modelo AAA originais foram compradas no mercado formal enquanto as falsificadas no mercado informal ("camelódromo"). As baterias, original e falsificada, são da mesma marca e modelo (Figura 1).

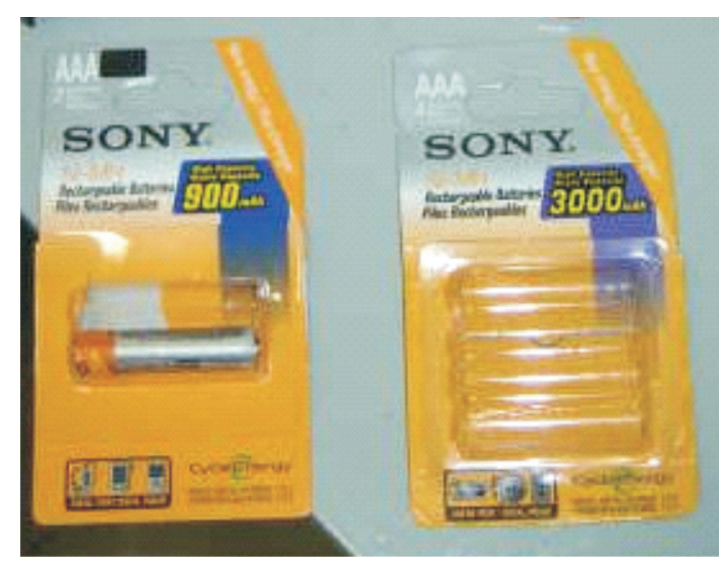

Figura 1. Embalagens das baterias original (esquerda) e falsificada (direita).
O desempenho elétrico (capacidade de carga e potência elétrica) foi obtido de ciclos cronopotenciométricos e a composição com auxílio das técnicas de espectroscopia de fluorescência de Raios X (EDXRF) e difração de Raios X (XRD). Foi caracterizado ainda o design das baterias e a massa dos seus eletrodos, uma vez que esses parâmetros também influenciam no desempenho elétrico da bateria (WEN-HUA et al., 1995; DENG et al., 1999; ZHOU et al., 1999; CHEN, 2001; ABEA et al., 2006; WANGA et al., 2005; JIANXIN et al., 2001; LIU et al., 2004).

As capacidades de carga e as potências elétricas das baterias foram investigadas realizando-se ciclos de carga e descarga sob corrente controlada (DURACELL, 2009; PANASONIC, 2005; GUA et al., 1999). No processo de carga foi aplicada uma corrente elétrica de $0,1 \mathrm{C}$ pelo tempo de $16 \mathrm{~h}$, que equivale a uma sobrecarga de $60 \%(\mathrm{C}=$ capacidade de carga nominal dividido por hora $=\mathrm{mAh} / \mathrm{h}) . \mathrm{Em}$ baixas correntes $(0,1 \mathrm{C})$ a bateria de Ni-HM pode ser sobrecarregada, sem danos aos seus eletrodos, para garantia de carregamento pleno.

$\mathrm{Na}$ descarga aplicou-se uma corrente $0,2 \mathrm{C}$, para que o tempo de descarga da bateria fosse de $5 \mathrm{~h}$ (PANASONIC, 2005; LINDEN; REDDY, 2002). Experimentos exploratórios mostraram que a bateria original carregada a $0,1 \mathrm{C}(90 \mathrm{~mA})$ e descarregada a 0,2 C (180 mA) comporta-se dentro do esperado, enquanto a bateria falsificada carregada a $0,1 \mathrm{C}$ (300 $\mathrm{mA})$ foi drasticamente sobrecarregada $\mathrm{e}$ quando descarregada a $0,2 \mathrm{C}(600 \mathrm{~mA})$ sua tensão atinge o limite de 1,0 V instantaneamente. Para se estabelecer a real capacidade de carga da bateria falsificada, variou-se a corrente elétrica até que o tempo de descarga fosse de $5 \mathrm{~h}$. Isso foi conseguido com corrente de 30,8 mA. Esta corrente equivale portanto à taxa de $0,2 \mathrm{C}$.

A capacidade de carga (em módulo) é obtida multiplicando-se a corrente aplicada pelo tempo de aplicação. A potência fornecida pelas baterias foi obtida, multiplicando-se a tensão, da curva de 
descarga (tensão vs. tempo), pelo valor da corrente aplicada $(0,2 \mathrm{C})$. Os ensaios elétricos foram realizados num ciclador de baterias da marca Flyever, com dois canais simultâneos de análise e corrente máxima de $1 \mathrm{~A}$, interfaciado a um microcomputador. O design interno das baterias foi investigado desmontando seu invólucro metálico com micro serra rotativa numa capela com exaustão de ar. Após o corte as baterias foram submetidas à vácuo para secagem do eletrólito. A composição química dos eletrodos das baterias foi determinada, após preparação dos mesmos na forma de pó, com auxílio de duas técnicas, a Fluorescência de RaiosX por Dispersão em Energia (EDXRF - Energy Dispersive X-ray Fluorescence) e a Difratometria de Raios X (XRD - X-ray Diffraction). A EDXRF foi realizada com excitação de radiação Ag K $\alpha$ (MOXTEK FT 100), detetor Si-PIN (AMPTEK XR 100CR), tempo de aquisição de $500 \mathrm{~s}$, tensão de $28 \mathrm{kV}$, corrente de $10 \mu \mathrm{A}$, e com as amostras acomodadas em filme de poliéster (CHEMPLEX). A XRD foi feita num difratômetro X'Pert PRO MRD Philips, com radiação $\mathrm{Cu} \mathrm{K} \alpha$ na geometria focalizante BraggBrentano, tensão de $40 \mathrm{KV}$ e corrente de $50 \mathrm{~mA}$. O intervalo de varredura $2 \theta$ utilizado foi de $15^{\circ}$ a $90^{\circ}$, com passo angular de $0,025^{\circ}$ e tempo de contagem de $4 \mathrm{~s}$ por ponto. A massa dos eletrodos foi medida numa balança Snug, modelo 150, com 0,005 g de precisão.

\section{Resultados e discussão}

A Figura 2 apresenta a relação entre as medidas da tensão e capacidade de carga para as baterias, original e falsificada obtidas das curvas de carga e descarga das baterias. No carregamento a corrente é constante e positiva (por convenção), e a capacidade de carga é uma função crescente no tempo. Na descarga a corrente também é constante, mas negativa (por convenção), e a capacidade de carga (ou de descarga) é uma função decrescente no tempo. No ponto A, de ambas as curvas da Figura 2, está localizado o final da carga (com sobrecarga) e o início da descarga. Observa-se na região de sobrecarga uma constância no valor da tensão da bateria original $(\mathrm{dV} / \mathrm{dt} \cong 0)$ contra um decréscimo da tensão da bateria falsificada $(\mathrm{dV} / \mathrm{dt}<0)$. Esta inflexão negativa na curva de tensão é típica de baterias de Ni-Cd mesmo carregadas sob baixas correntes de 0,1C (LINDEN; REDDY, 2002). Nota-se, para ambas baterias, que nem toda a carga inserida é extraída. Isso é comum tanto para baterias de Ni-HM como de Ni-Cd, uma vez que no processo de sobrecarga à $0,1 \mathrm{C}(\sim 92 \mathrm{~mA}$ para a original e $\sim 15,4$ mA para a falsificada) ocorrem várias reações de oxirredução sobrepondo-se à reação reversível de armazenamento de carga.

No descarregamento (processo espontâneo) dois valores de capacidade de carga puderam ser obtidos das curvas de descarga, um na tensão de $1,2 \mathrm{~V}\left(\mathrm{Q}_{1,2 \mathrm{~V}}\right)$ e outro na tensão de $1,0 \mathrm{~V}\left(\mathrm{Q}_{1,0 \mathrm{~V}}\right)$. $\mathrm{O}$ valor $\mathrm{Q}_{1,2 \mathrm{~V}}$ será confrontado com a capacidade de carga fornecida no rótulo da bateria, uma vez que a capacidade de carga lá informada, para ambas as baterias, referese capacidade medida na descarga até a tensão 1,2 V. A tensão de 1,0 V é uma tensão mínima e típica para os sistemas Ni-HM e Ni-Cd, que certifica a descarga plena das baterias, e que neste trabalho foi utilizada como base para o cálculo das taxas $\mathrm{C}$ de carga e descarga. As capacidades de carga obtidas para a bateria original foram: $\mathrm{Q}_{1,2 \mathrm{~V}}=750 \mathrm{mAh}$ e $\mathrm{Q}_{1,0 \mathrm{~V}}=920 \mathrm{mAh}$, enquanto para a falsificada as capacidades foram: $\mathrm{Q}_{1,2 \mathrm{~V}}=130 \mathrm{mAh}$ e $\mathrm{Q}_{1,0 \mathrm{~V}}=154$ $\mathrm{mAh}$. O custo benefício desta bateria ao invés de 1,6 R\$/Ah é, portanto, de $38 \mathrm{R} \$ / \mathrm{Ah}$.

A potência das baterias à $0,2 \mathrm{C}$ e no tempo médio de análise $(2,5 \mathrm{~h})$ foi de $220 \mathrm{~mW}$ para a bateria original e de $41 \mathrm{~mW}$ para a falsificada. Já sob uma maior corrente de descarga, de $0,8 \mathrm{C}$, que equivale a $736 \mathrm{~mA}$ para a bateria original e $123 \mathrm{~mA}$ para a bateria falsificada. As potências obtidas, no tempo médio de descarga, foram respectivamente, $840 \mathrm{~mW}$ e $140 \mathrm{~mW}$. Tanto a bateria original como a falsificada foram completamente descarregadas na corrente $0,2 \mathrm{C}$ em $5 \mathrm{~h}$. Já à $0,8 \mathrm{C}$ a bateria original levou $1 \mathrm{~h}$ e $20 \mathrm{~min}$ e a falsificada $12 \mathrm{~min}$. Isso indica 
que a bateria falsificada exibe perda de capacidade de carga em altas correntes que pode ser atribuída a fatores intrínsecos como baixa condutividade eletrônica dos seus eletrodos, baixa difusibilidade iônica $\left(\mathrm{H}^{+}\right)$no eletrólito e eletrodos, entre outros. Ressalta-se que o desempenho de potência elétrica da bateria falsificada descarregada a $0,8 \mathrm{C}$ é inferior ao desempenho da bateria original descarregada a $0,2 \mathrm{C}$.

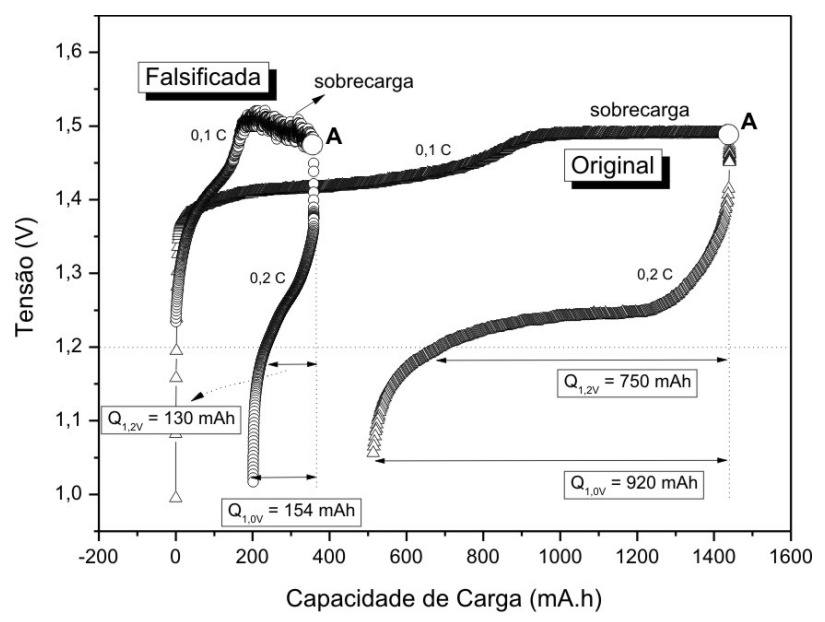

Figura 2. Tensão vs. capacidade de carga das baterias original e falsificada.

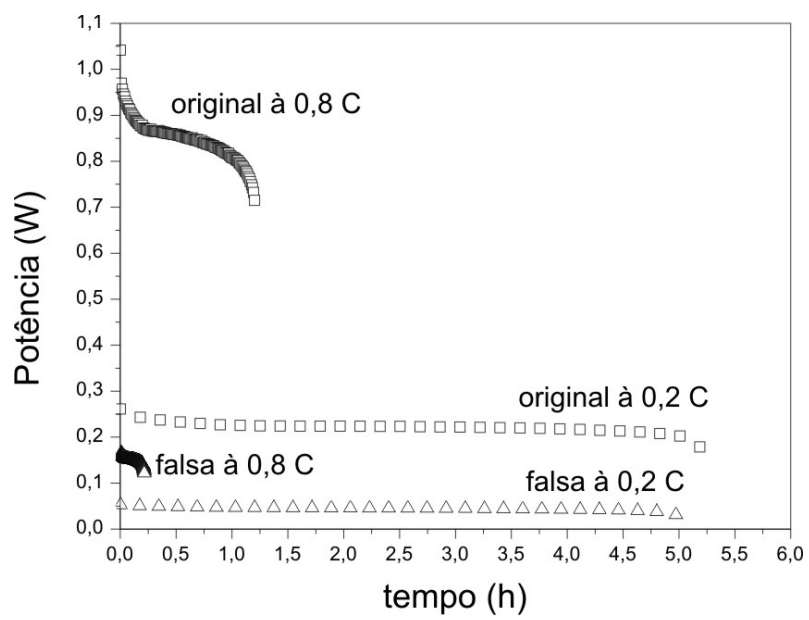

Figura 3. Curvas de potência vs. tempo das baterias, original e falsificada, sob correntes de $0,2 \mathrm{C}$ e $0,8 \mathrm{C}$.

As baterias no estado descarregado foram desmontadas e observou-se que ambas possuem o mesmo design, tipo rocambole ou "jelly roll". Os catodos tem a mesma textura, e estão suportados numa matriz de níquel metálico sinterizado. O material eletroativo dos eletrodos negativos recobrem um mesmo tipo de fita metálica que possui $0,2 \mathrm{~mm}$ de espessura e simetricamente perfurada. As massas dos catodos das baterias, original e falsificada, foram respectivamente 4,64 e 2,40 g, enquanto para os anodos, original e falsificado, as massas foram respectivamente de 2,60 e 1,19 g, ou seja, os eletrodos da bateria original possuem aproximadamente o dobro da massa dos eletrodos da bateria falsificada. Ambas as baterias são encapsuladas em tubo cilíndrico de aço inoxidável. Apesar do baixo desempenho apresentado pela bateria falsificada, chama a atenção o nível tecnológico com que estas baterias são construídas, possuindo design, suporte de eletrodo e contatos elétricos dignos de uma bateria de alta qualidade.

As análises de EDXRF revelaram que nos catodos é predominante a presença de níquel (Figura 4a). Já para os anodos os espectros indicam a presença de terras raras $(\mathrm{TR}=\mathrm{La}, \mathrm{Ce}, \mathrm{Pr}, \mathrm{Nd}, \mathrm{Pm})$ e níquel para a bateria original, e de cádmio e níquel para a bateria falsificada (Figura 4b).

Os difratogramas de raios-X revelaram para ambos os catodos, a presença predominante do composto $\mathrm{Ni}(\mathrm{OH})_{2}$, além de carbono grafite e níquel metálico presentes no catodo falsificado (Figura 5a). Já os difratogramas de raios-X dos anodos (Figura 5b), revelam que a liga $\mathrm{LaNi}_{5}$ e níquel metálico (Ni) estão presentes na composição do anodo original, enquanto que no anodo falsificado foi encontrado o composto $\mathrm{Cd}(\mathrm{OH})_{2}$ (Figura 5b). Alguns picos do difratograma do anodo da bateria falsificada não puderam ser identificados, apesar das muitas tentativas. Todas as fases encontradas nos difratogramas de raios-X (Fig.5a e Fig.5b), estão em concordância com os elementos indentificados pelas medidas de EDXRF. 

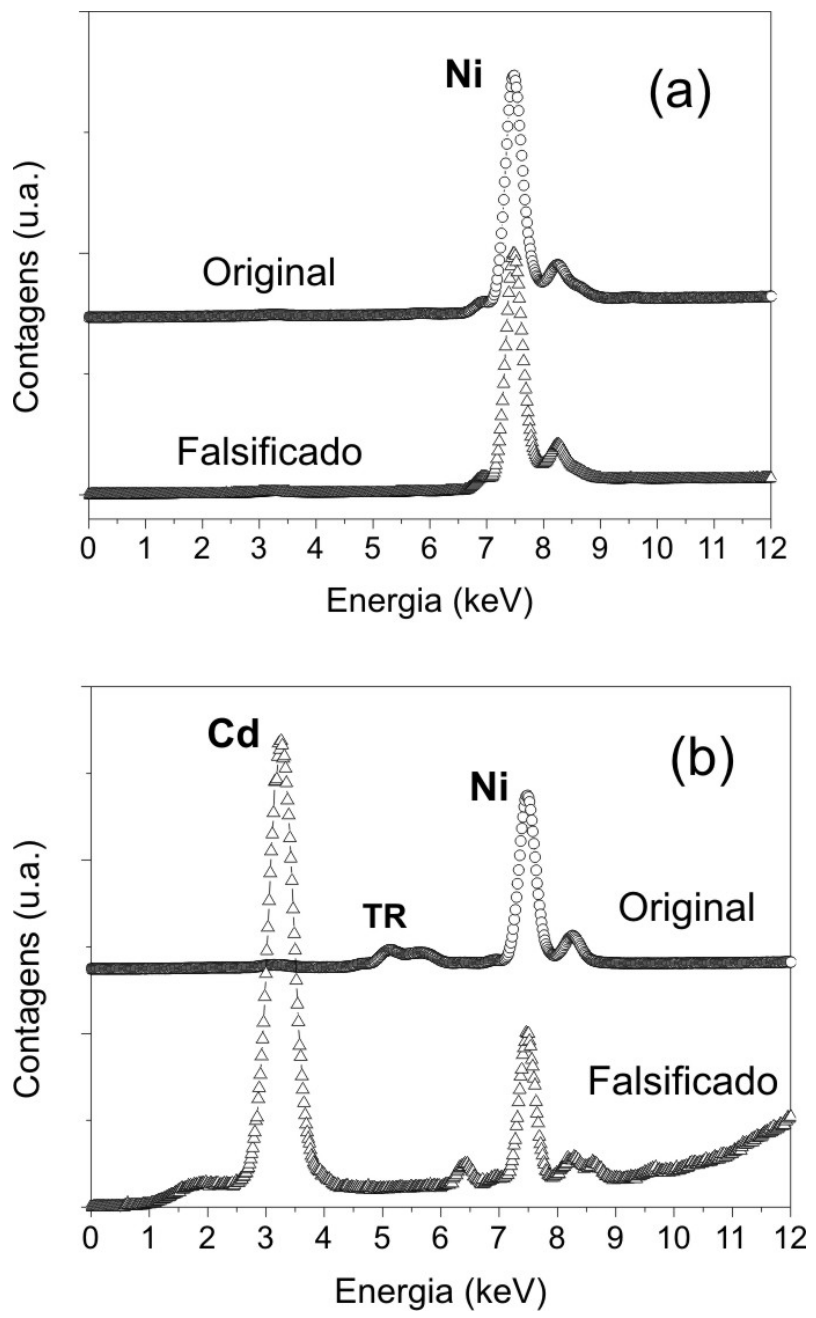

Figura 4. Espectros de EDXRF dos catodos (a) e anodos (b) das baterias original e falsificada.

Um resumo geral comparando as capacidades de carga, as potências sob diferentes correntes, o design, a composição química predominante e a
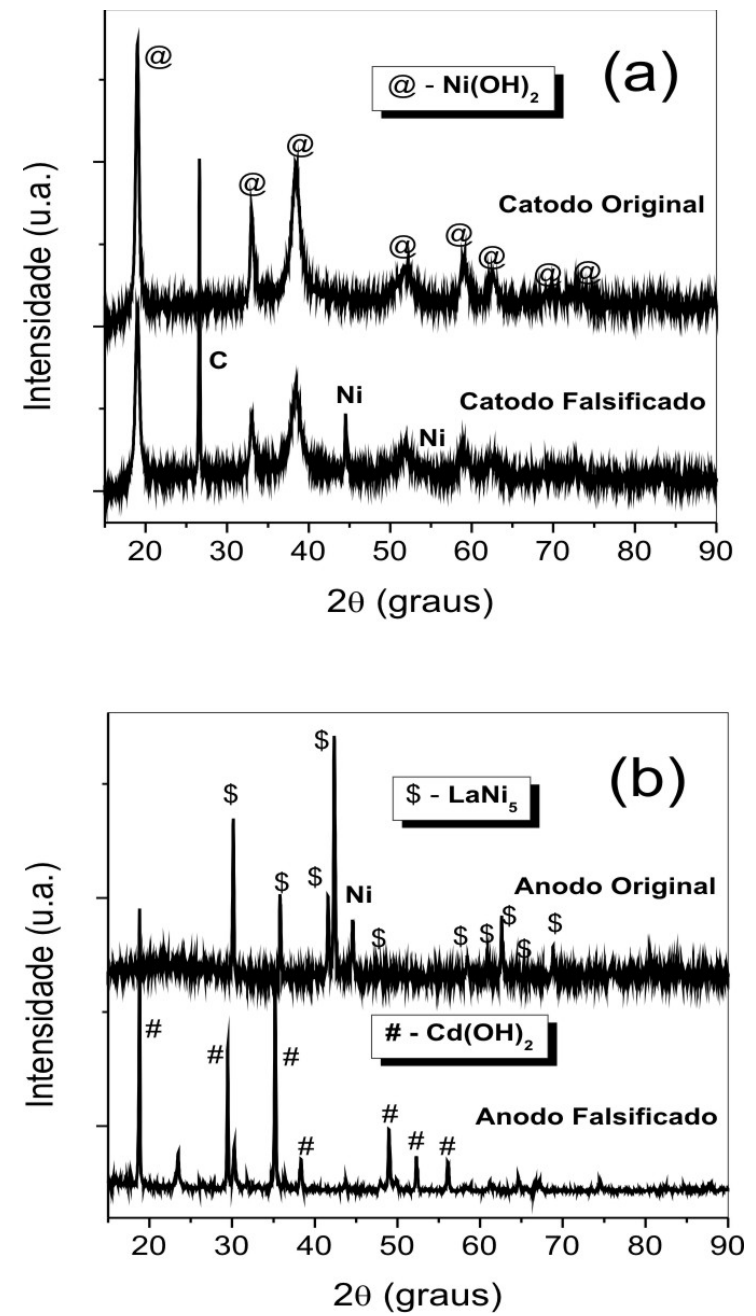

Figura 5. Difratogramas de raios $X$ dos catodos (a) e anodos (b) das baterias, original e falsificada.

massa dos eletrodos e a relação custo/benefício para os dois tipos de baterias são apresentados na Tabela 1 . 
Tabela 1 - Dados comparativos entre as baterias, original e falsificada.

\begin{tabular}{lll}
\hline PARÂMETROS & ORIGEM DA BATERIA & \\
\hline & Falsificada & Original \\
\cline { 2 - 3 } Custo R\$ & 5,00 & 15,00 \\
Informações do Rótulo & $3000 \mathrm{mAh}, 1,2 \mathrm{~V}$ & $900 \mathrm{mAh}, 1,2 \mathrm{~V}$ \\
Capacidade de Carga medida a 1,2 V & $130 \mathrm{mAh}$ & $750 \mathrm{mAh}$ \\
Capacidade de Carga medida a 1,0 V & $155 \mathrm{mAh}$ & $920 \mathrm{mAh}$ \\
Potência à 0,2 C (mW) & 41 & 220 \\
Potência à 0,8 C (mW) & 140 & 840 \\
Design & $j e l l y ~ r o l l$ & $j e l l y ~ r o l l$ \\
Composição & $\mathrm{Ni}(\mathrm{OH})_{2}(+), \mathrm{Cd}(\mathrm{OH})_{2}(-)$ & $\mathrm{Ni}(\mathrm{OH})_{2}(+), \mathrm{LaNi}_{5}(-)$ \\
Massa Eletrodo (+) & $2,40 \mathrm{~g}$ & $4,64 \mathrm{~g}$ \\
Massa Eletrodo (-) & $1,19 \mathrm{~g}$ & $2,60 \mathrm{~g}$ \\
Capacidade Específica (mAh/g) & $42 \mathrm{mAh} / \mathrm{g}$ & $127 \mathrm{mAh} / \mathrm{g}$ \\
Custo Benefício (Rótulo) & $1,6 \mathrm{R} \$ / \mathrm{Ah}$ & $16 \mathrm{R} \$ / \mathrm{Ah}$ \\
Custo Benefício (Real) & $38 \mathrm{R} \$ / \mathrm{Ah}$ & $20 \mathrm{R} \$ / \mathrm{Ah}$ \\
\hline
\end{tabular}

\section{Conclusões}

Neste trabalho, baterias de Ni-HM do formato AAA, original e falsificada, foram analisadas quanto às suas capacidades de carga, potência em diferentes correntes de descarga, design de construção, massa e composição dos eletrodos.

Com relação às informações fornecidas nos rótulos, ambas as baterias não cumprem com o descrito nos rótulos. A bateria original é de fato de Ni-HM, mas a capacidade de carga indicada no rótulo (900 mAh) só foi conseguida para a tensão limite de 1,0 V. Para tensão limite de 1,2 V a capacidade de carga da bateria original foi de 750 $\mathrm{mAh}$. O custo versus benefício elétrico desta bateria deve, portanto, ser corrigido para $20 \mathrm{R} \$$ /Ah ao invés dos $16 \mathrm{R} \$ / \mathrm{Ah}$, devido ao fato que muitos aparelhos eletrônicos necessitam de $1,2 \mathrm{~V}$ de tensão mínima para operarem. Todas outras informações estão dentro do esperado para este tipo de bateria.

A bateria falsificada informa em seu rótulo ser de Ni-HM e exibir uma capacidade de carga de 3000
mAh. Foi verificado que estas informações estão incorretas, ou seja, o anodo não é de hidreto metálico e sim de hidróxido de cádmio e a capacidade de carga não ultrapassa os 154 mAh.

A capacidade de carga específica $(\mathrm{mAh} / \mathrm{g})$ da bateria original é muito superior à da bateria falsificada o que significa que o baixo desempenho elétrico da bateria falsificada não pode ser atribuído simplesmente à menor massa dos seus eletrodos (duas vezes menor). Analisando-se a relação do custo vs. benefício de carga "específica" $(\mathrm{R} \$$ $(\mathrm{mAh} / \mathrm{g}))$, conclui-se não haver vantagem entre os tipo de baterias, sendo de $126 \mathrm{R} \$(\mathrm{mAh} / \mathrm{g})$ para a falsificada e de $127 \mathrm{R} \$ /(\mathrm{mAh} / \mathrm{g})$ para a original. Por outro lado, o consumidor ao comprar uma bateria está comprando capacidade de carga (mAh), ou seja, uma unidade que, grosso modo, se refere à duração no fornecimento de energia elétrica, e não capacidade de carga específica $(\mathrm{mAh} / \mathrm{g})$, que se refere à duração no fornecimento por peso.

Outra vantagem exibida pela bateria original, 
em detrimento à falsificada, foi a potência de operação. As potências da bateria falsificada nas taxas de 0,2 ou $0,8 \mathrm{C}$ são menores do que a da bateria original à $0,2 \mathrm{C}$. Isso significa que muitos aparelhos eletrônicos, principalmente os baseados na tecnologia de semicondutores e de comunicação, não poderão operar com as baterias falsificadas devido ao fato desses equipamentos necessitarem de uma potência mínima para operação.

$\mathrm{O}$ fato do anodo da bateria falsificada ser à base de cádmio, ao invés de um hidreto metálico, implica num perigoso agente de contaminação ambiental, uma vez que ao ser rotulada como sendo de Ni-HM, pode ser depositada no lixo doméstico (CONAMA, 1999). Os processos de coleta e reciclagem também são seriamente prejudicados, pois, não há como identificar, de maneira rápida e barata, a real composição dos eletrodos da baterias.

Em suma, as baterias falsificadas lesam o consumidor tanto pelo seu desempenho elétrico como ambiental.

\section{Agradecimentos}

À Sercomtel Celular S.A. e à Fundação Araucária pelo apoio financeiro e à Capes pelas Bolsas de Mestrado.

\section{Referências}

ABEA, H.; MORIMOTO, R.; SATOH, F.; AZUMAB, Y.; UCHIDA, H. Effects of ion irradiation on hydriding rate of Mm based hydrogen storage alloy. Journal of Alloys and Compounds, Lausanne, v. 408/412, p. 348350, 2006.

AZAR, C.; HOLMBERG, J.; LINDGREN, K. Methodological and ideological options, socioecological indicators for sustainability. Ecological Economics, Amsterdam, v. 18, n. 2, p. 89-112, 1996.

BUCHMANN, I. Batteries in a portable world: a handbook on rechargeable batteries for non-engineers. New York: Cadex Electronics, 2001.
CHEN, W. Effects of surface treatments of MINi4.0 Co0.6Al0.4 hydrogen storage alloy on the activation, charge/discharge cycle and degradation of $\mathrm{Ni} / \mathrm{MH}$ batteries. Journal of Power Sources, Lausanne, v. 92, n. 1/2, p. 102-107, 2001.

CONSELHO NACIONAL DO MEIO AMBIENTE CONAMA. Resolução $n^{\circ}$. 257, de 30 de junho de 1999. Disponível em: <http://www.mma.gov.br /port/conama/ res/ res99/ res25799.html>. Acesso em: 7 dez. 2009.

COSTA, F. D. Com prazo contando, setor de pilhas ainda não organizou sistema de coleta de usadas. Revista Sustentabilidade, São Paulo, 3 ago. 2009. Disponível em: <http://www. Revistasustentabilidade .com.br/ reciclagem/ falta-de-coleta- seletiva- atrapalhaprogramas- de-reciclagem- de-pilhas- e-baterias /? searchterm $=$ pilhas $>$. Acesso em: 7 dez. 2009.

DENG, B.; LI, Y.; WANG, R.; FANG, S. Two reduction processes for hydrogen adsorption and absorption at MmNi5-type alloy electrodes. Electrochimica Acta, New York, v. 44, n. 17, p. 2853-2857, 1999.

DURACELL. Ni-MH recheargeable batteries. Disponível em: <http://www.duracell.com/oem/Pdf/ others/TECHBULL.pdf $>$. Acesso em: 7 dez. 2009.

FENGA, F.; GENGA, M.; NORTHWOODB, D. O. Electrochemical behaviour of intermetallic-based metal hydrides used in $\mathrm{Ni}=$ metal hydride $(\mathrm{MH})$ batteries: a review. International Journal of Hydrogen Energy, Oxford, v. 26, n. 7, p. 725-734, 2001.

GENG, M.; FENG, F.; GAMBOA, S. A.; SEBASTIAN, P. J.; MATCHETT, A. J.; NORTHWOOD, D. O. Electrocatalytic characteristics of the metal hydride electrode for advanced Ni-MH batteries. Journal of Power Sources, Lausanne, v. 96, n. 1, p. 90-93, 2001.

GUA, W. B.; WANGA, C. Y.; LIB, S. M.; GENGC, M. M.; LIAWC, B. Y. Modeling discharge and charge characteristics of nickel metal hydride batteries. Electrochimica Acta, New York, v. 44, n. 25, p. 45254541, 1999.

HUGGINS, R. A.; PRINZ, H.; WOHLFAHRTMEHRENS, M.; JIIRISSEN, L.; WITSCHEL, W. Proton insertion reactions in layered transition metal oxides. Solid State Ionics, Amsterdam, v. 70/71, part. 1, p. 417424, 1994.

JIANXIN, M.; PAN, H.; ZHU, Y.; LI, S.; CHEN, C. Electrochemical properties of La0.9Sm0.1 Ni(5.0_x $)$ Cox (x 2.0, 2.5, 3.0) hydride electrode alloys. Electrochimica Acta, New York, v. 46, N. 15, p. 2427-2434, 2001.

LINDEN, D.; REDDY, T. B. Handbook of batteries. New York: McGraw-Hill, 2002. 
LIU, Y.; PAN, H.; GAO, M.; ZHU, Y.; LEI, Y.; WANG, Q. The electrochemical performance of a $\mathrm{La}-\mathrm{Mg}-\mathrm{Ni}-$ Co-Mn metal hydride electrode alloy in the temperature range of 20 to 30C. Electrochimica Acta, New York, v. 49, n. 4, p. 545-555, 2004.

MORIOKAA, Y.; NARUKAWAB, S.; ITOUB, T. Stateof-the-art of alkaline rechargeable batteries. Journal of Power Sources, Lausanne, v. 100, n. 1/2, p. 107-116, 2001.

OHMS, D.; KOHLHASE, M.; BENXZUR-URMOSSY, G.; SCHADLICH, G. New Developments on high Power alkaline batteries for industrial applications. Journal of Power Sources, Lausanne, v. 105, n. 2, p. 127-133, 2002.

PANASONIC. Nickel metal hydride batteries. 2005. Disponível em: <http:// www. panasonic.com/ industrial /battery/ oem /images/pdf/ Panasonic_NiMH_Overview. pdf>. Acesso em: 7 dez. 2009.

PANASONIC. Nickel metal hydride batteries. 2005. Disponível em: <http:// www. panasonic. Com / industrial /battery/oem /images/pdf /Panasonic NiMH ChargeMethods.pdf $>$. Acesso em: 7 dez. 2009.

RYDH, C. J.; BOSVARD, A. B. "Impact on global metal flows arising from the use of portable rechargeable batteries". Science of the Total Environment, Amsterdam, v. 302, n. 1/3, p. 167-184, 2003.

SAKAI, T.; UEHARA, I.; ISHIKAWA, H. R\&D on metal hydride materials and $\mathrm{Ni}-\mathrm{MH}$ batteries in Japan. Journal of Alloys and Compounds, Lausanne, v. 293-295, p. 762769, 1999.

SHUKLAA, A. K.; VENUGOPALANB, S.; HARIPRAKASHA, B. Nickel-based rechargeable batteries. Journal of Power Sources, Lausanne, v. 100, n. $1 / 2$, p. 125-148, 2001.

WANGA, Y.; GAOA, X. P.; LUA, Z. W.; HUB, W. K.; ZHOUA, Z.; QUA, J. Q.; SHENA, P. W. Effects of metal oxides on electrochemical hydrogen storage of Nanocrystalline LaMg12-Ni composites. Electrochimica Acta, New York, v. 50, n. 11, p. 2187-2191, 2005.

WEN-HUA，Z.; JIA-JUN, K. E.; HONG-MEI, Y.; DENG-JUN, Z. A study of the electrochemistry of nickel hydroxide electrodes with various additives. Journal of Power Sources, Lausanne, v. 56, n. 1, p. 75-79, 1995.

YAN, D.; CUI, W. Preparation and properties of nobinder electrode Ni/MH battery. Journal of Alloys and Compounds, Lausanne, v. 293/295, p. 780-783, 1999.

YUICHI, S.; SHIGEO, T.; KOICHI, K. Cause of the memory effect observed in alkaline secondary batteries using nickel electrode. Journal of Power Sources, Lausanne, v. 93, n. 1/2, p. 20-24, 2001.

ZHOU, Z. Q.; LIN, G. W.; ZHANG, J. L.; GE, J. S.; SHEN, J. R. Degradation behavior of foamed nickel positive electrodes of Ni-MH batteries. Journal of Alloys and Compounds, Lausanne, v. 293-295, p. 795-798, 1999.

Recebido em 23 Dezembro, 2009 - Received on December 23, 2009

Aceito em 13 Maio, 2010 - Accepted on May 13, 2010. 
